\title{
How Important is Computing Technology for Library and Information Science Research?1
}

Mike Thelwall, Nabeil Maflahi

Computers in Library and Information Science (LIS) research have been an object of study or a tool for research for at least fifty years, but how central are computers to the discipline now? This article analyses the titles, abstracts and keywords of forty years of articles in LIS-classified journals for trends related to computing technologies. The proportion of Scopus LIS articles mentioning some aspect of computing in their title, abstract or keywords increased steadily from 1986 to 2000, then stabilising at about two thirds, indicating a continuing dominance of computers in most LIS research. Within this general trend, many computer-related terms have peaked and then declined in popularity. For example, the proportion of Scopus LIS article titles, abstracts or keywords that included the terms computer or computing decreased fairly steadily from about $20 \%$ in $\mathbf{1 9 7 5}$ to $5 \%$ in 2013 and the proportion explicitly mentioning the web peaked at $18 \%$ in 2002. Parallel analyses suggest that computing is substantially less important in two related disciplines: education and communication, and so it should be seen as a key aspect of the LIS identity.

\section{Introduction and background}

The recent history of LIS research has clearly been affected by computers, from computing systems in libraries to the rise of the personal computer and the web. Nevertheless, no study has attempted to quantify just how great this influence is, although there have been investigations into the history of computers, the history of LIS research, and even the history of computers in LIS research. Histories of computing sometimes start with the abacus (Ifrah, Harding, Bellos, \& Wood, 2000) or mass automation techniques, such as the commercial mechanical calculating machines of over a century ago (e.g., Campbell-Kelly \& Aspray, 2004). Research and development for electronic calculating machines and stored program computers was boosted significantly by the urgent code-breaking needs of the Second World War and the post-war years saw the rise of a small number of expensive flexible computers in research organisations and large companies. As power, reliability and the availability of trained technicians increased, computers started to be used more widely in the business and government sectors. A revolutionary transformation occurred when it became possible to build small personal computers, making them much cheaper and therefore capable of taking on an increasing range of office tasks. It also became practical for individuals to own their own computer at this stage. The next revolution was the development of the web and the infrastructure to deliver it via the internet through phone lines, and then to smart devices (Bergman, Gradovitch, Bar-Ilan, \& Beyth-Marom, 2013), creating the globally interconnected ubiquitous information and entertainment world of today.

Although libraries have been important since ancient times (Lerner, 2009), LIS formally entered higher education in the form of a School of Library Economy in the USA in 1884 which was part of a small international movement towards systematising library education at the time (White, 1976). The core ideas of information science were then

\footnotetext{
${ }^{1}$ Thelwall, M. \& Maflahi, N. (in press). How important is computing technology for library and information science research? Library and Information Science Research.
} 
developed within the Institute of Bibliography in Brussels from 1895, although not using modern terminology (Rayward, 1997). The term library science was apparently popularised much later by a widely influential book from India, The Five Laws of Library Science (Ranganathan, 1931) and the term information science was only coined in 1955 (Shapiro, 1995). In the USA, library science extended from a focus on libraries to a wider focus on information and this was reflected by the almost universal extension of school names to LIS, starting in 1964 (Galvin, 1977). This change occurred in parallel with a shift in focus from documents to the wider concept of information, which was at least partially driven by the emergence of new technologies (Borko, 1968; Buckland, 1996). The shift was also probably influenced by attempts to automate searching for documents (Allen, 1962), leading to the development of information retrieval as a specialism. This specialism was boosted from 1992 with US Department of Defense and National Institute of Standards and Technology sponsorship the Text REtrieval Conference (TREC) series. The importance of information was then further underlined by the US-centred information school (iSchool) organization from 2005 (http://ischools.org/about/), often losing the term library altogether from school names. A number of previous studies have tracked influential topics within LIS using citations (Garfield, 2009) and co-citations (Åström, 2007), showing that it contains distinct subfields, each with their own history, and that it has important influences from other disciplines (Cronin, 2008; Tang, 2004).

Sustained LIS interest in computers probably started with the recognition that they could automate many of the core labour-intensive, information-based functions of libraries, such as those concerned with cataloguing and lending books (Rayward, 2002). Since LIS is often about organising information and helping users to find relevant information, any system that could efficiently store data in a searchable way is potentially useful for libraries and also for wider information science research. Electronic computers were first investigated for their potential uses in libraries as far back as the 1950s (Borgman, 1997; Kilgour, 1970), and related research directions, such as analysing the logs of search systems, started in the 1960s (Peters, 1993), if not before. Almost from the start, computers did not just automate the existing functions of libraries but also extended them (Rayward, 2002), potentially leading to sustained interest in research into computer-based innovations for libraries and for the wider information work of LIS professionals. As early as 1974, Rosenberg (1974) claimed that computers were so central to information science that they have become a core part of its "way of looking at the world" so that information scientists may think in computer processing terms even for actions taken by computers. This approach has been developed by Warner (2008), who suggests that information technology is influential on theoretical discourses of information science but is essentially seen as external rather than an integral part of them.

The importance of computing to some but not all LIS is suggested by various studies of the field. For example, some of the 11 coherent research topics of the 120 most prolific authors in terms of published information science research 1972-1995 were about computing technologies (experimental retrieval, online retrieval, general library systems, OPACs), some probably had computers as an important aspect (citation analysis, bibliometrics, science communication) but others did not (user theory, indexing theory, citation theory, communication theory). A later study of 2002-2004 covering four general LIS journals and the specialist journal Scientometrics (28\%) (Janssens, Leta, Glänzel, \& De Moor, 2006) found four out of five computing-related clusters (bibliometrics (2), webometrics, information retrieval, patent analysis) and one partially-related cluster (social issues, 
combining computing terms like internet with others like seek, behaviour, health, and social). This shift towards more computing-related research in LIS and a strong web influence (also in: Åström, 2007) was confirmed by results from 1996-2005, although the later study found 2 topics that do not seem to be directly related to computing out of the 12 analysed (user theory, structured abstracts; also to some extent children's information searching behaviour and users' judgements of relevance) (Zhao \& Strotmann, 2008). The situation in 2006-2010 was similar, with only one out of 12 factors seeming to be unrelated to computers (knowledge management), although it could be argued that computing technology had started to become part of the background of, rather than core to, other topics found, including research evaluation, information behaviour, bibliometrics, bibliometric distributions and perhaps even technology acceptance (Table 3 in: Zhao \& Strotmann, 2014). This latter issue highlights the problem that research topics relying upon computers, such as most bibliometrics, at some stage may rarely find the development of computer technologies to be directly relevant to research goals and so computers may become part of the background of the area rather than key to driving new research.

\section{Problem statement}

A number of bibliometric studies have shown that computing or web-related topics have been important to LIS at specific points in time (see above) but there is no evidence about how important or central computing technologies are in LIS research. A previous article has used a bibliometric approach to analyse the whole of LIS over 100 years, tracking changes in the use of keywords in article titles over time and found many computing-related keywords to increase and then decrease in frequency (Larivière, Sugimoto, \& Cronin, 2012) but did not analyse computing overall as a single entity. The current article fills this gap with an analysis of the extent to which computers, the web and computing are explicitly mentioned in LIS journal articles. Although almost all modern research probably uses computers, from word processing papers to analysing statistics with software, most uses are not important enough to be mentioned in the published article. For example, it would be strange to report that the current article was written in Microsoft Word or that the graphs were drawn by Microsoft Excel. In contrast, research is sometimes about an aspect of computing (e.g., designing or evaluating hardware or software). Moreover, aspects of computing technology can also be important enough to be mentioned in the title, keyword or abstract of research that is not primarily about computing. Such mentions may occur, for example, if technology makes the research possible even though the study is not about it. This article is based upon the simplifying assumptions that if a computing technology is mentioned in the title of an article then it is likely to be central to the article and if it is mentioned in the title, abstract or keywords then it is likely to be important in some way for the article. The following research questions drive the investigation.

- What is the proportion of LIS articles that computing is important for, and how has this changed over time?

- What is the proportion of LIS articles that computing is central to, and how has this changed over time?

- How does LIS compare to similar disciplines in terms of the importance of computing?

- Which topics have fallen in and out of fashion in computing related and noncomputing-related LIS research over the past 40 years? 
Answering these questions can give a fuller understanding of the relative importance of computing that can help the discipline to plan the amount of computing that should be contained in its educational curricula, debate the merits of the current balance of computing-related research and predict the likely relevance of computing in the future.

\section{Methods}

The overall strategy is to analyse a large sample of titles, abstracts and keywords from LIS journal articles and to calculate the yearly percentage of articles containing computerrelated terms in order to identify increases and decreases over time. Journal articles were chosen rather than the full range of scholarly outputs (e.g., conference papers, monographs, book chapters) in order to focus on a clearly defined type of output that seems to be the most important for a majority of the LIS discipline and is well-covered by available databases. Monographs and book chapters are probably important for areas of LIS with a humanities orientation but it does not seem possible to gain a large sample of these for the subject area in an unbiased way. The largest current source is probably Google Books (e.g., Chen 2012) and it is possible to conduct bibliometric analyses with this database (Kousha, Thelwall, \& Rezaie, 2011) but it contains no pre-classified set of LIS books to select from. The Book Citation Index in the Web of Science is also a large source of books and book chapters as far back as 2005 (Torres-Salinas, Rodríguez-Sánchez, Robinson-Garcia, Fdez-Valdivia, \& García, 2013) but this is not enough for the current study. Conference papers are also presumably important for areas of LIS that are close to computer science (e.g., Goodrum, McCain, Lawrence, \& Lee Giles, 2001). Although conference papers are classified in some databases and hence could be included, they seem to be less important than journal articles in most LIS areas and so excluding them should give clearer results that only focus on (a proportion of) primary outputs.

The most practical source of LIS journal articles is a major international scholarly article index and there are many possible alternatives, none of which are comprehensive (Meho, \& Spurgin, 2005). Scopus was selected because it seems to have at least as good coverage as WoS and the coverage of WoS seems to be close to the best of the available sources for LIS (Meho, \& Spurgin, 2005). A recent previous study has shown that WoS and Scopus give similar results for a range of bibliometric measures (Archambault, Campbell, Gingras, \& Larivière, 2009) and so an equivalent WoS study would presumably give similar findings.

The titles, abstracts and keywords of all Scopus papers of type journal article from the Library and Information Sciences subfield were downloaded. The 50 journal titles with the most articles were inspected to check that they were appropriate for the LIS category and the following seven titles were excluded for inappropriate content: Telecommunications Policy; The Scientist; Opera Quarterly; Notes and Queries; Early Music; Computers and Education; IEEE Transactions on Information Theory. The last of these titles was initially retained for its focus on information but it showed up as an anomaly in the results and a closer examination revealed it to have little to do with LIS. Although some of the journals cover information systems rather than LIS these were kept because these two subject areas are often classified together (e.g., in Scopus, WoS and the UK RAE) and so it seems reasonable to accept their inclusion within LIS. The Scopus LIS category is not exhaustive because some LIS journals are coded into computing and other categories. For example the Journal of the American Society for Information Science and Technology was excluded for all years except 2001-8. Only articles with a title and abstract in Scopus were used. Many early 
articles did not have an abstract and including such articles would give a false impression of changes over time. The coverage of Scopus is also low before 1996 (Li, Burnham, Lemley, \& Britton, 2010), which adds to this problem. After these steps, 58,513 articles were included in the study.

A list of computing terminology was needed to identify computer-related articles in the data set. A simple approach would be just to use the term computer and synonyms but more detailed information could be obtained from a more comprehensive list. For example, articles on particular types of computer might mention a specific name rather than the generic term. Ideally, the list of terms should include all historical terms (e.g., floppy disk) as well as current terms. A complicating factor is polysemy: some terminology can refer to either an aspect of computing or something unrelated to computing (e.g., apple, mouse). To resolve this, the Free Online Dictionary Of Computing (FOLDOC, foldoc.org) was chosen as a large academic-related source of computing terms. This resource has been maintained since 1985 and therefore serves as a source of historical as well as contemporary computerrelated terms. FOLDOC contains 14,943 different terms, including single words, numbers and phrases. As a practical step, only single word terms were considered, reducing the number to 8,802 . This list was then intersected with the list of terms extracted from all article titles, keywords and abstracts downloaded, leaving a total of 3,620 relevant computing terms. Some of these terms also had non-computing meanings, such as concatenate and erotica. This list was therefore manually filtered by the first author to remove terms that did not always relate to computing in the article titles, keywords and abstracts downloaded. This produced a smaller list of 761 terms. Inspection of the articles matching none of these terms resulted in an additional seven words being added: e-access; e-government; electronic; computer-aided; inspec; online; kerberos. The collection of articles was then split into two parts, with the computing-related articles (the accuracy of this term is discussed later) being those that contained at least one of the 768 words in their abstract, title or keywords and the remaining articles being those containing none of the terms. This and the remaining data analysis was conducted using the free software Mozdeh (http://mozdeh.wlv.ac.uk).

The Scopus LIS category is not necessarily an accurate reflection of the LIS field, even with the clearly out of scope journals removed. In addition, there is no clear dividing line between LIS and non-LIS research. For example, scientometrics and chemoinformatics are specialisms that are both taught in some LIS schools but there may be disagreement on whether these are clearly within LIS. An alternative strategy to monitor the importance of computing in LIS research would be to analyze the core journals of the discipline and ignore the rest. This has the advantage that the contents of the core journals would be widely agreed to be predominantly LIS but has the disadvantage that LIS areas that are specialized enough to launch their own journal (e.g., scientometrics, informetrics, chemoinformatics) would presumably be underrepresented in general journals as a result of the alternative outlets. Nevertheless, results from this alternative perspective would be useful to test the robustness of the results from the larger data set and so a second analysis was conducted for a set of four mainstream LIS journals: Journal of the American Society for Information Science and Technology (JASIST) (and alternative names), Journal of Documentation (JDoc), Library and Information Science Research (LISR), and Information Processing and Management (IP\&M).

The inclusion of a word in the title or keywords of an article presumably indicates that it is more important than if it is mentioned only in the abstract, and perhaps this is an 
indicator that the word is likely to be central to the text. Hence parallel analyses were conducted of article titles and keywords. For these analyses, all articles without abstracts were excluded (for comparability with the main analyses since many early articles didn't have abstracts) even though the abstracts were ignored.

It is useful to differentiate between general computing-related trends in research and trends that are specific to LIS. To help with this, parallel analyses were conducted of two other Scopus categories, Education and Communication. Both of these professional social sciences fields have some overlap with LIS and so are suitable for comparison purposes.

Graphs were produced of the proportion of computing-related articles mentioning the term computer or computing to track changes in the use of terms most directly referencing computers. Similar graphs were also produced for the term Internet and terms directly referring to the web (web or WWW).

In order to identify LIS topics that changed in importance over time, the time period was split into four approximately equal segments, 1974-1983, 1984-1993, 1994-2003 and 2004-2013, separately for the computing-related articles and the remaining articles. For each word in the abstract (excluding copyright and publisher statements), title and keyword list of each article, the proportion of articles containing that word was calculated in each of the four time periods. Following this, a difference in proportions test $z$ value was calculated for each word to assess the difference in proportions for that word between each consecutive time period. These results were used to produce a list of the terms with the most substantial increase or decrease between successive time periods. These lists were manually filtered by the first author to remove terms not alluding to a research topic, theme or method (e.g., we, paper, article, method) as well as computing-related terminology in the non-computing set (e.g., TREC) and the ten words with the most substantial increases and decreases were reported (as measured by the difference in proportions $z$ value).

\section{Results and discussion}

Scopus includes abstracts for LIS articles starting from 1975 (except for four earlier articles, which were ignored) and the number of indexed articles increased steadily from then, with a rapid increase from 2005 (Figure 1). Most of the increase is due to indexing a larger number of journals rather than increases in the sizes of individual journals and due to the start date of Scopus (Li, Burnham, Lemley, \& Britton, 2010). 


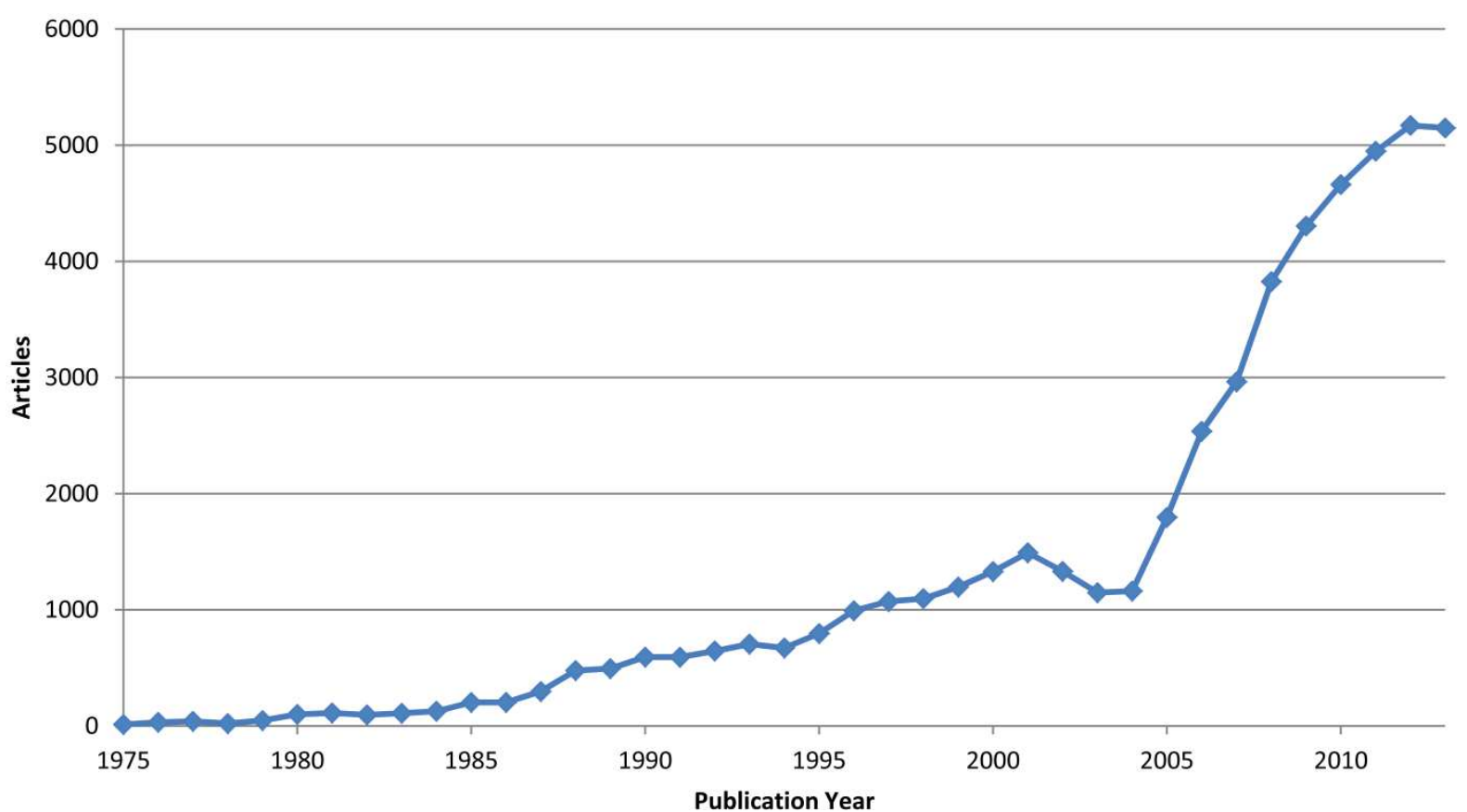

Figure 1. The number of Scopus LIS articles with abstracts published in each year in the period 19752013 (journals categorized as LIS, excluding 7 journals with out-of-category content).

\section{The importance of computing to LIS research}

A mention of a term in the title, keyword or abstract of an article seems likely to be a reasonable indicator that the term is important to the article, although irrelevant words can be included for creative purposes (e.g., "Is interindexer consistency a hobgoblin" is not about mythical creatures). The converse is a bit more tentative: an important concept may be discussed through synonyms, meronyms, or hyponyms of the main terms and so the absence of a term does not necessarily imply that it is unimportant to an article. To guard against these issues, random samples of articles were checked to ensure that the overall proportions of articles with computing terms were approximately correct in figures 2 and 3 . Using Excel's random number generator a random sample of 100 articles classified as computing and a random sample of 100 articles classified as not computing were selected from the year 2013 for both data sets (i.e., 400 altogether). The 200 texts for each dataset were arranged in a random order and blind coded by the first author for explicit mentions of computing. For the title, abstract and keywords data set (Figure 2), 80\% of the articles automatically classified as explicitly mentioning computing by the above method were also human coded as explicitly mentioning computing and $74 \%$ of the articles automatically classified as not explicitly mentioning computing were also human coded as not explicitly mentioning computing. The corresponding figures for the title and keyword dataset were $85 \%$ and $74 \%$. These results suggest that whilst the automatic identification of explicit mentions of computing was only about $80 \%$ correct, this has probably not substantially affected the overall results in terms of the heights of the "Any computing word" lines in figures 2 and 3.

The proportion of LIS articles mentioning at least one computing term in their title, abstract or keywords increased steadily from the early 1980s until about 2000 and then has remained steady at about $65 \%$ (Figure 2 ). The proportion of articles mentioning computing terms may have increased since 2000 rather than remaining steady if highly specialist terms were increasingly mentioned, such as the names of specialist programs that would not be in 
FOLDOC (e.g., Mozdeh). Explicit mentions of the terms computer, computers or computing have steadily declined to about $5 \%$, however, presumably because more specialist terms have replaced them - perhaps a variant of the obliteration by incorporation phenomenon (e.g., McCain, 2012). In contrast, mentions of the Internet and web (or www) peaked in 2002 and have declined steadily since, probably also due to replacement with more specialist terms.

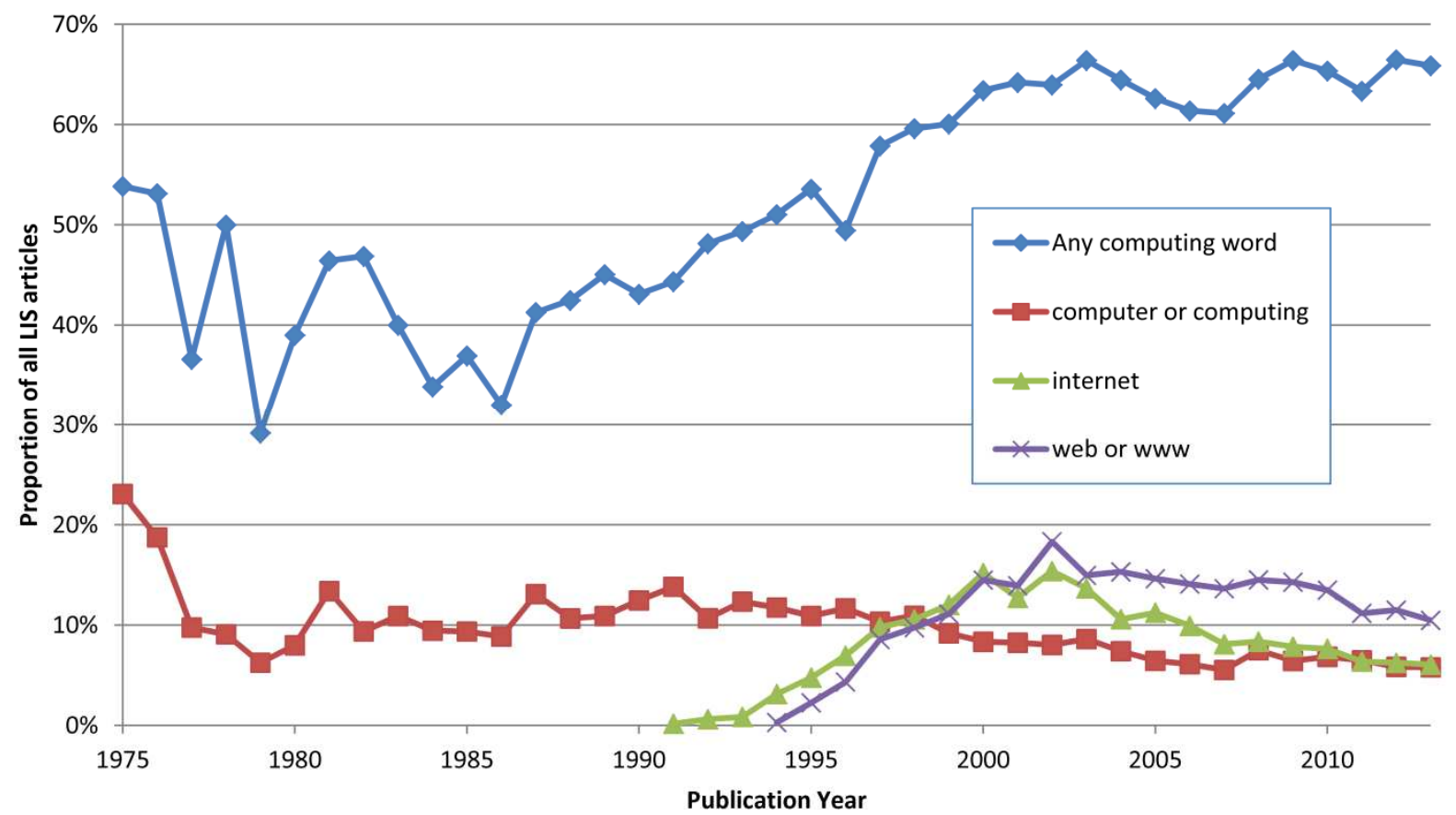

Figure 2. The proportion of Scopus LIS articles with abstracts 1975-2013 according to the terms in their titles, abstracts or keywords (journals categorized as LIS in Scopus, excluding 7 journals with out-of-category content - see methods).

The equivalent results for the four mainstream journals (Figure 3 ) are broadly similar. The main differences are a higher percentage of computing-related articles up to the year 2000, perhaps due to the computing-oriented IP\&M, and a higher percentage of web-related articles from the year 2000 to 2010, perhaps due to the field of Webometrics, which published mainly in JDoc, JASIST, IP\&M, and Scientometrics, or due to the rise of Google and blogs. 


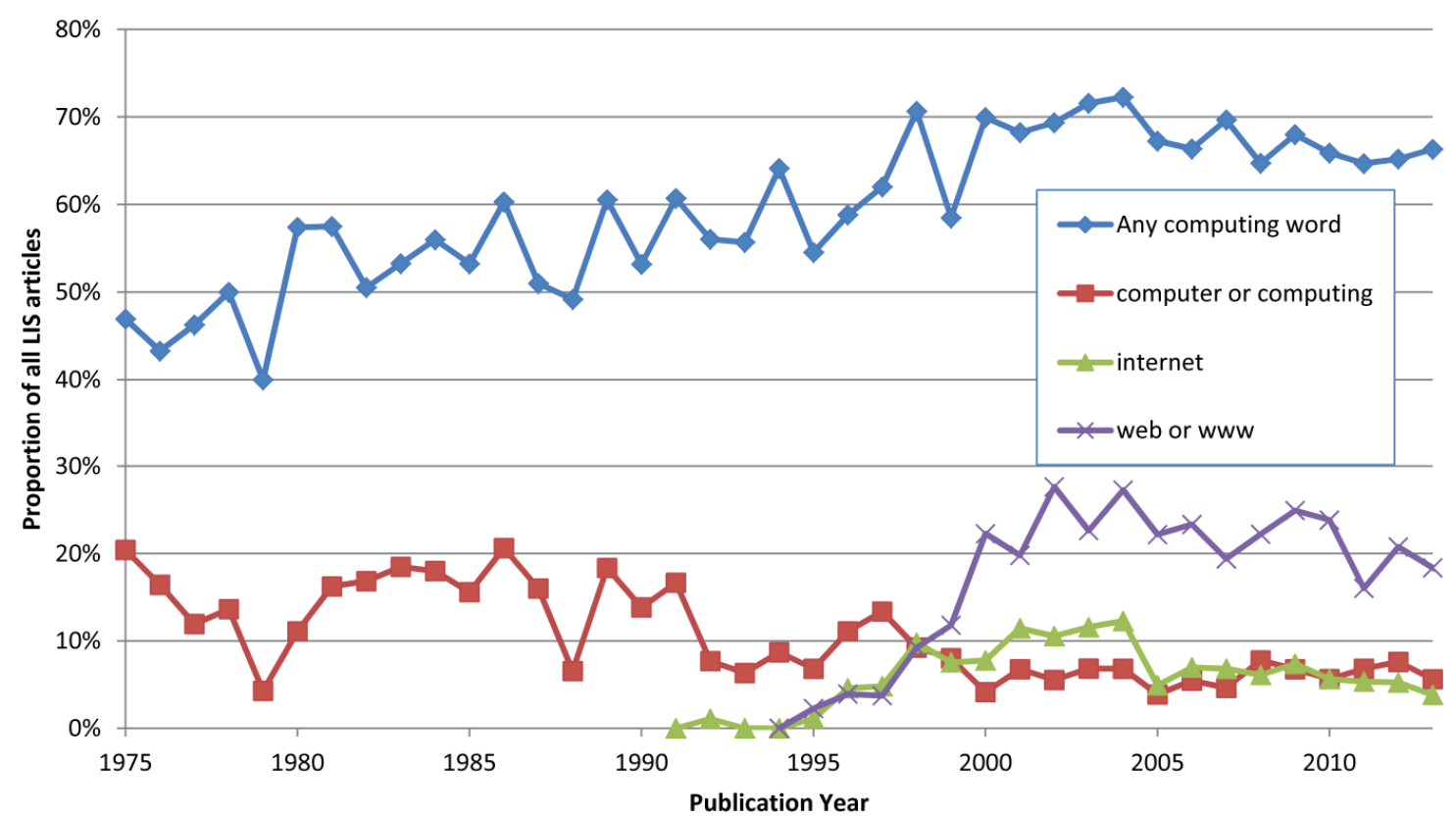

Figure 3. The proportion of Scopus articles from LISR, JASIST, JDoc and IP\&M with abstracts 1975-2013 according to the terms in their titles, abstracts or keywords.

\section{The centrality of computing to LIS research}

A mention of a term in the title or keyword list of an article seems likely to be a reasonable indicator that the term is central to the article, rather than just being important to it, although all the provisos in the paragraph above apply to this case. Figure 4 suggests that the proportion of articles for which computing was central increased steadily until the year 2000 and then levelled off at $40-45 \%$. It shows a similar trend for the other three lines, except that it indicates that these topics are central to (i.e., mentioned in the keywords or title) about half of the articles for which they are important (i.e., mentioned in the keywords, abstract or title), and this peaks at about $10 \%$ of LIS articles in each case.

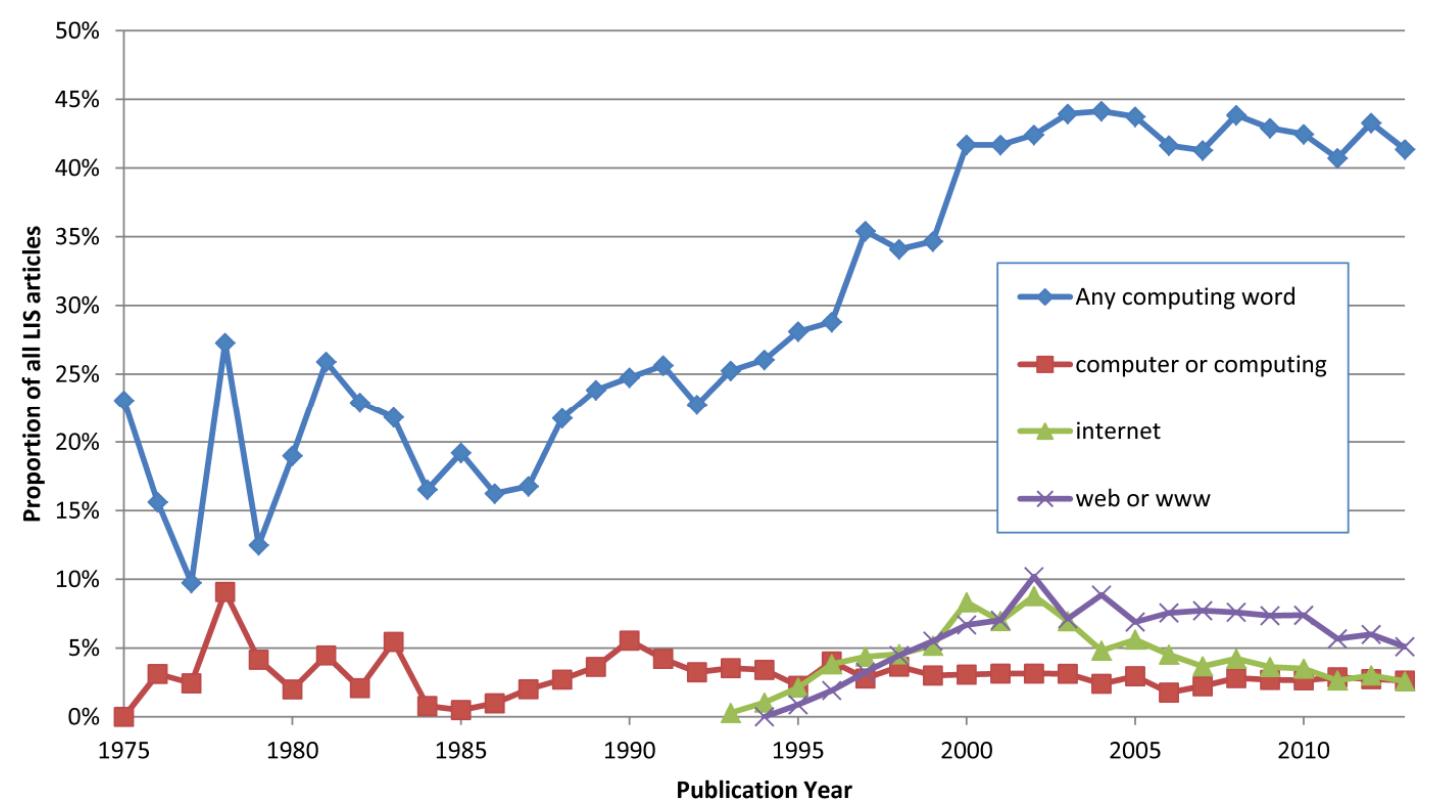

Figure 4. The proportion of Scopus LIS articles with abstracts 1975-2013 according to the terms in their titles or keywords (journals categorized as LIS in Scopus, excluding 7 journals with out-of-category content- see methods). 
The results for the four general journals (Figure 5) are again similar except for the higher amount of web-related research.

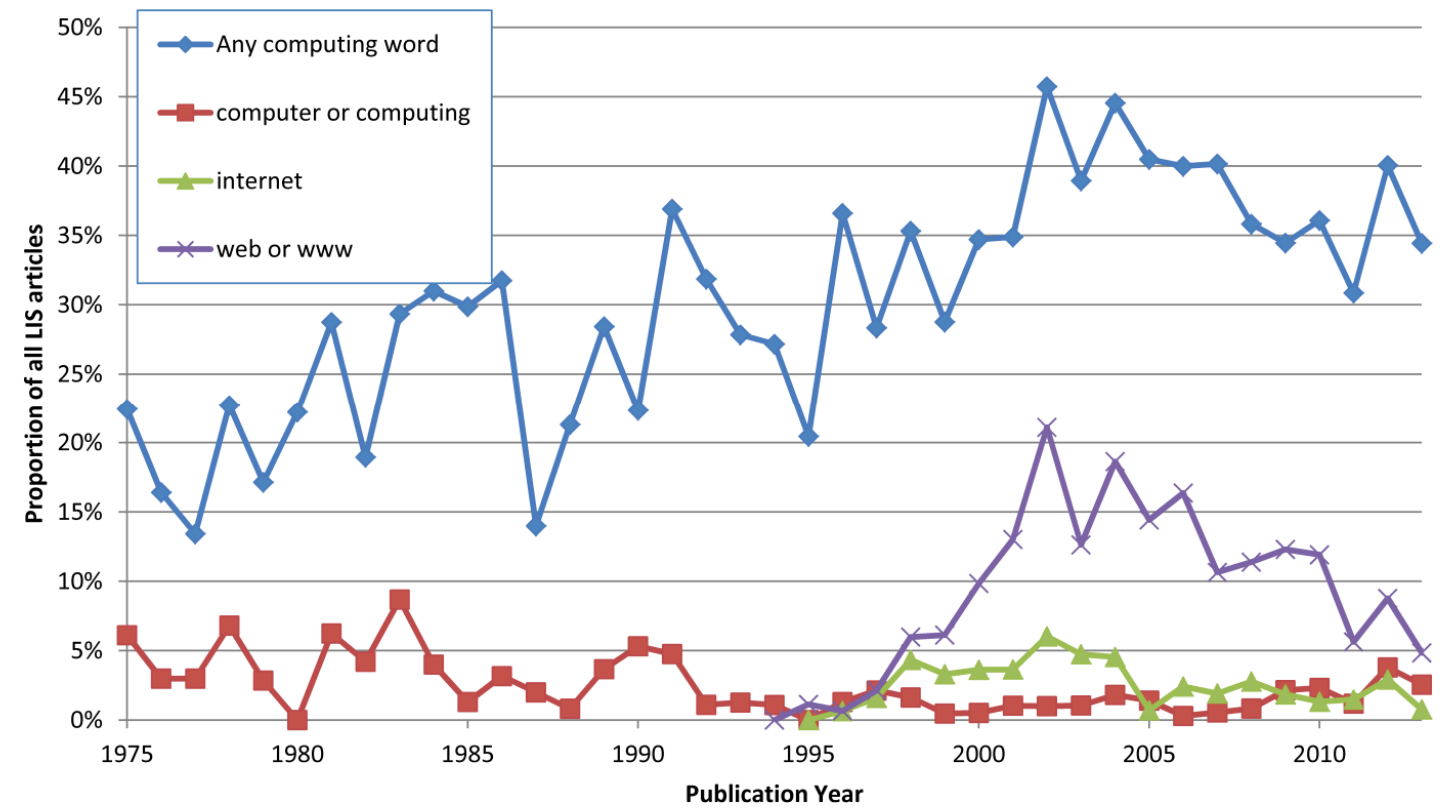

Figure 5. The proportion of Scopus articles from LISR, JASIST, JDoc and IP\&M with abstracts 1975-2013 according to the terms in their titles or keywords.

\section{Computing in Education and Communication research}

The trends for Education (Figure 6) and Communication (Figure 7) are broadly similar to those for LIS, except for the Education decrease in 1996 (coinciding with a doubling of the number of articles indexed) and a Communication spike in 1995 (possibly due to computingrelated special issues) and the lower rate of inclusion of computing. Overall, computing appears to be substantially less important in research for these disciplines than for LIS.

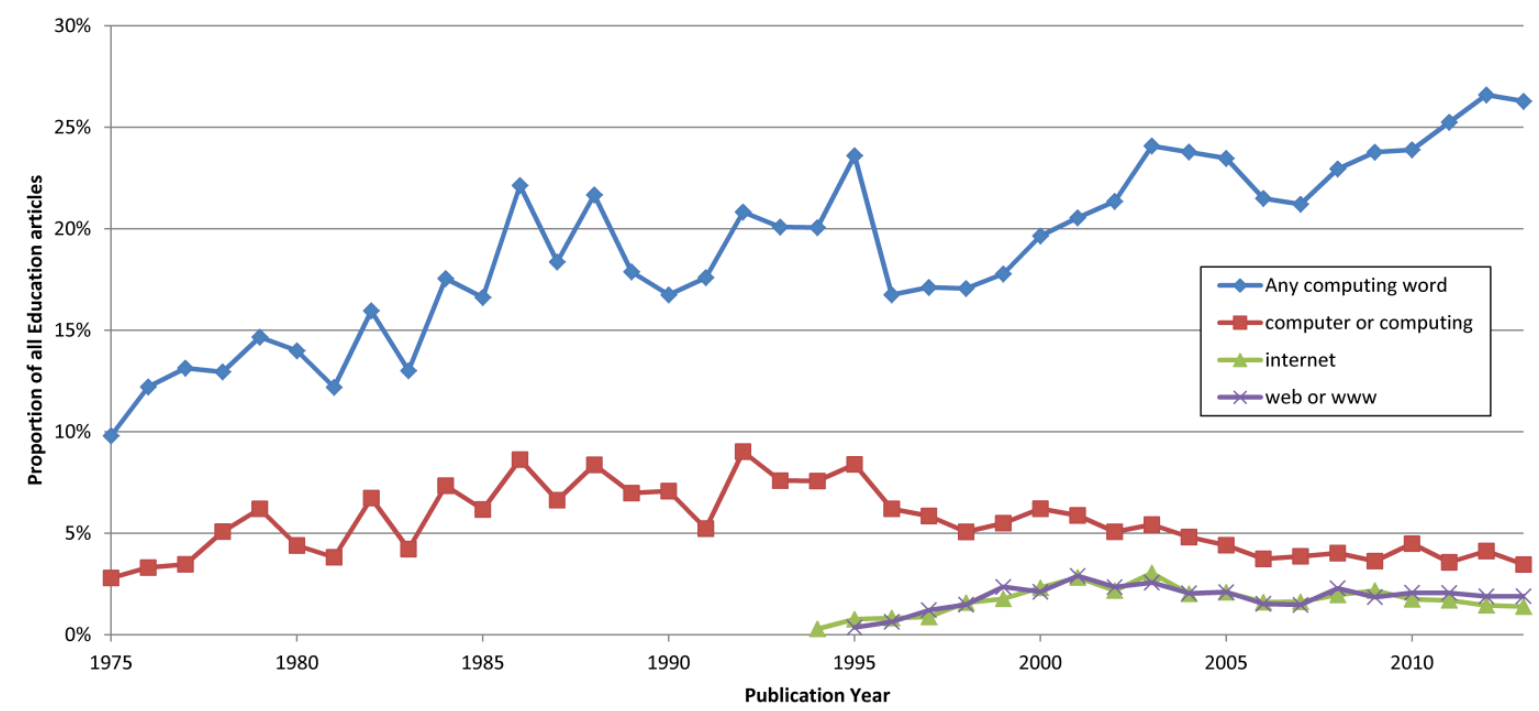

Figure 6. The proportion of Scopus Education articles with abstracts 1975-2013 according to the terms in their titles, abstracts or keywords (journals categorized as LIS in Scopus, excluding 7 journals with out-of-category content- see methods). 


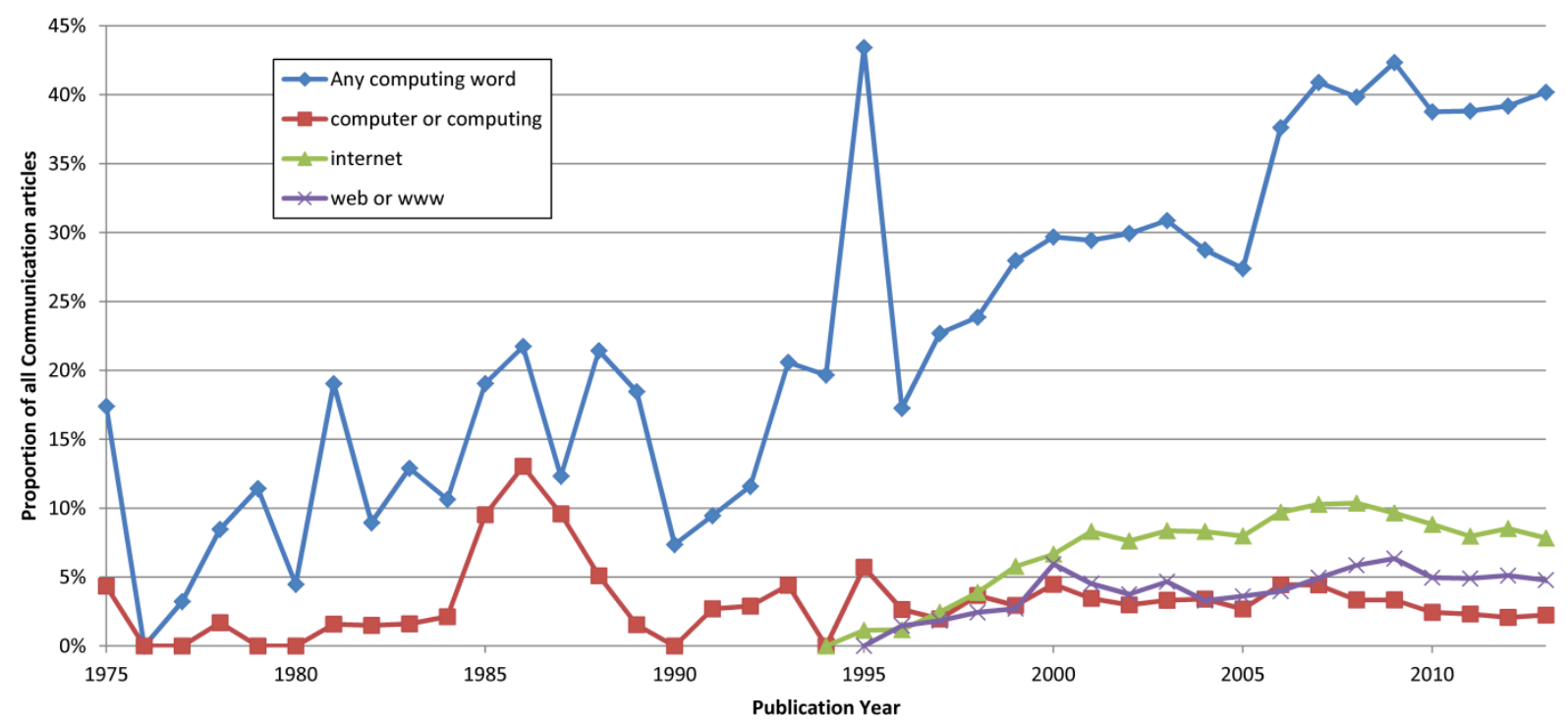

Figure 7. The proportion of Scopus Communication articles with abstracts 1975-2013 according to the terms in their titles, abstracts or keywords (journals categorized as LIS in Scopus, excluding 7 journals with out-of-category content - see methods).

\section{Fashionable topics in computing-related and non-computing-related LIS research}

Table 1 shows the words that have gone into and out of fashion for computing-related LIS research, at least in terms of being used in abstracts, titles and keywords. Some of these words relate to specific computing-related technologies that were created and then became obsolete or declined in use (e.g., CD-ROM, SGML, terminal), and were replaced with names of specific examples (e.g., hypertext by web, software by Google) or were replaced by synonyms (e.g., data-base with database; www and world wide web with web), or became ubiquitous enough that they did not need to be mentioned explicitly (e.g., software, computer, internet, electronic, online, file, automated, automatic, storage, processing). The cause of many of the new computing words in 2004-13 is the introduction of chemoinformatics journals in this period (e.g., molecular, compound, protein). This shows that adding individual specialist journals can greatly affect the results of this method. 
Table 1. Terms with the most substantial changes in relative frequency between consecutive time periods for the computing-related Scopus LIS articles (excluding 7 journals with out-ofcategory content - see methods) with abstracts 1975-2013 (+1 is biggest rise between periods, -1 is biggest fall between periods).

\begin{tabular}{|l|l|l|l|}
\hline Rank & $\mathbf{1 9 7 5 - 8 3}$ vs. 1984-93 & $\mathbf{1 9 8 4 - 9 3 ~ v s . ~ 1 9 9 4 - 0 3}$ & $\mathbf{1 9 9 4 - 2 0 0 3 ~ v s . ~ 2 0 0 4 - 1 3 ~}$ \\
\hline+1 & software & web & algorithm \\
\hline+2 & electronic & internet & Google \\
\hline+3 & CD-ROM & digital & simulation \\
\hline+4 & tool & resource & molecular \\
\hline+5 & media & world & mobile \\
\hline+6 & issue & site & compound \\
\hline+7 & expert & electronic & protein \\
\hline+8 & database & wide & open \\
\hline+9 & practice & learning & website \\
\hline+10 & technology & web-based & target \\
\hline & & & \\
\hline-10 & interlending & online & WWW \\
\hline-9 & data-base & storage & SGML \\
\hline-8 & square & computer & world \\
\hline-7 & division & file & retrieval \\
\hline-6 & dialog & processing & internet \\
\hline-5 & lending & automated & hypertext \\
\hline-4 & automatic & bibliographic & computer \\
\hline-3 & recall & database & wide \\
\hline-2 & retrieval & automation & electronic \\
\hline-1 & on-line & microcomputer & CD-ROM \\
\hline & & &
\end{tabular}

Table 2 shows the terms that have gone into and out of fashion in terms of importance for LIS research that is apparently not computing-related. Some of these are concerned with knowledge management (e.g., knowledge, management, KM), business or management (e.g., BPR, management, manager), government (e.g., Reagan, perhaps also British), libraries (libraries), books (CIP, textbook, book), publishing (publishing, publisher) archives (archive, archival, archivist), mathematics (stationary, fuzzy, mathematical, weight, function) and education (education, student, learning, literacy). Whilst most of these terms may not primarily relate to computing, most probably have important computing aspects. For example, within knowledge management there is research into knowledge management systems (Sedighi \& Jalalimanesh, 2014). There are also a few computing-related terms (file, telecommunication) and at least one term that may be used predominantly in a computing context (retrieval; perhaps also technology). Overall, it seems that mathematical LIS declined in 1984-93, books and publishing declined in 1994-2003; and technology declined in 2004-13. 
Table 2. Terms with the most substantial changes in relative frequency between consecutive time periods for the Scopus LIS articles with abstracts 1975-2013 (excluding 7 journals with out-of-category content - see methods) that are not computing-related ( +1 is biggest rise between periods, -1 is biggest fall between periods).

\begin{tabular}{|l|l|l|l|}
\hline Rank & $\mathbf{1 9 7 4 - 8 3}$ vs. 1984-93 & $\mathbf{1 9 8 4 - 9 3}$ vs. 1994-03 & $\mathbf{1 9 9 4 - 2 0 0 3 ~ v s . ~ 2 0 0 4 - 1 3}$ \\
\hline+1 & librarian & knowledge & literacy \\
\hline+2 & management & collaboration & knowledge \\
\hline+3 & education & literacy & learning \\
\hline+4 & school & social & KM \\
\hline+5 & issue & business & sharing \\
\hline+6 & access & health & student \\
\hline+7 & manager & project & management \\
\hline+8 & role & learning & participant \\
\hline+9 & archive & context & h-index \\
\hline+10 & publishing & technology & performance \\
\hline & & & \\
\hline-10 & stationary & request & cellulose \\
\hline-9 & CIP & entitled & re-engineering \\
\hline-8 & hand & file & programme \\
\hline-7 & fuzzy & Reagan & BPR \\
\hline-6 & query & textbook & co-operation \\
\hline-5 & mathematical & publishing & S\&T \\
\hline-4 & weight & book & reengineering \\
\hline-3 & readily & archivist & British \\
\hline-2 & function & archival & telecommunication \\
\hline-1 & retrieval & publisher & technology \\
\hline & & & \\
\hline
\end{tabular}

\section{Limitations}

An important limitation of this study is its focus on core LIS journals in Scopus. It seems likely that LIS journals outside Scopus will tend to be less computing-related, especially if from less developed nations. The exclusion of conference papers probably serves to underestimate the influence of computing, since conference papers are important in computer science. This is counterbalanced to some extent by the exclusion of monographs and edited works, which presumably have a humanities orientation, with less computing.

The choice of LISR, JASIST, JDoc and IP\&M for the smaller-scale analysis is also a limitation because another set of journals with a different focus would have given different results. For example, Library Quarterly would also have been a reasonable choice, but may have included less computing and more library-focused research, and JASIST and IP\&M may tend to publish a relatively large proportion of computing-related research. Moreover, JASIST is a relatively large journal and its inclusion within the larger-scale analysis may skew the results towards computing research.

The reliance upon single terms rather than phrases has influenced some of the results. The main trend results are probably not affected, as shown by the manual crosschecking test of 200 articles (see above: The importance of computing to LIS research). Nevertheless, it has clearly affected tables 1 and 2 since these contain terms that have a different meaning in phrases (e.g., knowledge and management; world, wide and web). This 
exacerbates the existing problems, such as polysemy and the role of specialist journals. Hence tables 1 and 2 should only be taken as indicative and not definitive.

Another limitation is the choice of Education and Communication for comparisons with LIS because the differences found could be due to characteristics of the comparator fields rather than LIS. For example, computers may be irrelevant to large parts of educational research dealing with more theoretical issues (e.g., Yang, 2014).

\section{Conclusions}

Computing technologies are important to about two thirds of LIS research, at least in terms of published journal articles. This importance seems to have stabilised over the previous decade at about $65 \%$ but computers may be less frequently important for LIS research that is published in monographs and book chapters. Computers seems to be central for almost half of LIS research over the past decade, at least in terms of mentioning a computingrelated word in article titles or keyword lists. The increase and then stabilisation of importance of computing masks increases and subsequent decreases in mentions of specific technologies, with decreases often suggesting technological obsolescence or ubiquity. Despite this, there are aspects of LIS research for which computers seem to be often unimportant, such as knowledge management.

In terms of the methods used in this article, the technique for ranking words for the extent to which they increase or decrease in use between two different time periods was able to identify some trends and may be useful for future research into trends in academic subjects.

From a wider historical perspective, LIS has been concerned with finding efficient and effective ways to organise, search and store information for over a century and the widespread uptake and innovation with computers seems to be a natural progression. Because of this, at the moment it seems inconceivable that LIS researchers would one day cease to research and develop new computing technologies even when all current software and hardware have been replaced. Nevertheless, there seem to be some business, organisational, and governmental LIS research issues that are still not primarily about computing and so researchers should not assume that computing innovation will one day be a requirement for a successful LIS research career.

Finally, computer technologies seem to be more important for LIS research than for education and communication research, suggesting that computing is an important part of the LIS identity. This is presumably well-known but perhaps the extent to which this is true two thirds of current LIS research - may not be.

\section{References}

Archambault, É., Campbell, D., Gingras, Y., \& Larivière, V. (2009). Comparing bibliometric statistics obtained from the Web of Science and Scopus. Journal of the American Society for Information Science and Technology, 60(7), 1320-1326.

Åström, F. (2007). Changes in the LIS research front: Time-sliced cocitation analyses of LIS journal articles, 1990-2004. Journal of the American Society for Information Science and Technology, 58(7), 947-957.

Bergman, O., Gradovitch, N., Bar-Ilan, J., \& Beyth-Marom, R. (2013). Folder vs. tag preference in personal information management. Journal of the American Society for Information Science and Technology, 64(10), 1995-2012. 
Borgman, C. L. (1997). From acting locally to thinking globally: a brief history of library automation. The Library Quarterly, 215-249.

Borko, H. (1968). Information science: what is it? American Documentation, 19(1), 3-5.

Buckland, M. (1996). Documentation, information science, and library science in the USA. Information Processing \& Management, 32(1), 63-76.

Campbell-Kelly, M., \& Aspray, W. (2004). Computer: A History of the Information Machine (2 ed.). Boulder, CO: Westview Press.

Chen, X. (2012). Google Books and WorldCat: A comparison of their content. Online Information Review, 36(4), 507-516.

Cronin, B. (2008). The sociological turn in information science. Journal of Information Science, 34(4), 465-475.

Galvin, T. (1977). Pittsburgh. University of Pittsburgh Graduate School of Library and Information Sciences. In: A. Kent, H. Lancour \& J.E.Daily. (eds.) Encyclopedia of Library and Information Science (Vol. 22). New York: Marcel Dekker, Inc. (pp. 280-291).

Garfield, E. (2009). From the science of science to Scientometrics visualizing the history of science with HistCite software. Journal of Informetrics, 3(3), 173-179.

Goodrum, A. A., McCain, K. W., Lawrence, S., \& Lee Giles, C. (2001). Scholarly publishing in the Internet age: a citation analysis of computer science literature. Information Processing \& Management, 37(5), 661-675.

Ifrah, G., Harding, E. F., Bellos, D., \& Wood, S. (2000). The universal history of computing: From the abacus to quantum computing. New York: John Wiley \& Sons, Inc.

Janssens, F., Leta, J., Glänzel, W., \& De Moor, B. (2006). Towards mapping library and information science. Information Processing \& Management, 42(6), 1614-1642.

Kent, A. (1966). Textbook on mechanized information retrieval. New York: Interscience Publishers.

Kilgour, F. G. (1970). History of library computerization. Journal of Library Automation, 3(3), 218-229.

Kousha, K., Thelwall, M., \& Rezaie, S. (2011). Assessing the citation impact of books: The role of Google Books, Google Scholar, and Scopus. Journal of the American Society for Information Science and Technology, 62(11), 2147-2164.

Larivière, V., Sugimoto, C. R., \& Cronin, B. (2012). A bibliometric chronicling of library and information science's first hundred years. Journal of the American Society for Information Science and Technology, 63(5), 997-1016.

Lerner, F. (2009). The story of libraries: From the invention of writing to the computer age. New York, NY: Continuum.

Li, J., Burnham, J. F., Lemley, T., \& Britton, R. M. (2010). Citation Analysis: Comparison of Web of Science, Scopus, SciFinder, and Google Scholar. Journal of Electronic Resources in Medical Libraries, 7(3), 196-217.

McCain, K. W. (2012). Assessing obliteration by incorporation: Issues and caveats. Journal of the American Society for Information Science and Technology, 63(11), 2129-2139.

Meho, L.I. \& Spurgin, K.M. (2005). Ranking the Research Productivity of Library and Information Science Faculty and Schools: An Evaluation of Data Sources and Research Methods. Journal of the American Society for Information Science and Technology, 56(12), 1314-1331.

Peters, T. A. (1993). The history and development of transaction log analysis. Library Hi Tech, 11(2), 41-66.

Ranganathan, S. R. (1931). The five laws of library science. Madras: The Madras Library Association.

Rayward, W. B. (1997). The origins of information science and the International Institute of Bibliography/International Federation for Information and Documentation (FID). Journal of the American Society for Information Science, 48(4), 289-300.

Rayward, W. B. (2002). A history of computer applications in libraries: Prolegomena. IEEE Annals of the History of Computing, 24(2), 4-15.

Rosenberg, V. (1974). The scientific premises of information science. Journal of the American Society for Information Science, 25(4), 263-269. 
Sedighi, M., \& Jalalimanesh, A. (2014). Mapping research trends in the field of knowledge management. Malaysian Journal of Library \& Information Science, 19(1), 71-85.

Shapiro, F. R. (1995). Coinage of the term Information Science. Journal of the American Society for Information Science, 46(5), 384-385.

Tang, R. (2004). Visualizing interdisciplinary citations to and from information and library science publications. In Proceedings of Eighth International Conference on Information Visualisation. Los Alamitos: IEEE Press (pp. 972-977).

Torres-Salinas, D., Rodríguez-Sánchez, R., Robinson-Garcia, N., Fdez-Valdivia, J., \& García, J. A. (2013). Mapping citation patterns of book chapters in the Book Citation Index. Journal of Informetrics, 7(2), 412-424.

Warner, J. (2008). Organs of the human brain, created by the human hand? The social epistemology of information technology. Journal of Documentation, 64(5), 738-759.

White, H.D. \& McCain, K.W. (1998). Visualizing a discipline: an author co-citation analysis of information science, 1972-1995. Journal of the American Society for Information Science. 49(4), 327-355.

White, C. M. (1976). A historical introduction to library education: Problems and progress to 1951. Metuchen, NJ: The Scarecrow Press Inc.

Yang, H. C. (2014). Teaching sexual matters in Taiwan: the analytical framework for popular culture and youth sexuality education. Asia Pacific Journal of Education, 34(1), 49-64.

Zhao, D., \& Strotmann, A. (2008). Information science during the first decade of the web: An enriched author cocitation analysis. Journal of the American Society for Information Science and Technology, 59(6), 916-937.

Zhao, D., \& Strotmann, A. (2014). The knowledge base and research front of information science 2006-2010: An author cocitation and bibliographic coupling analysis. Journal of the Association for Information Science and Technology, 65(5), 995-1006.

\section{Appendix: Terms used to identify computing}

zip yahoo y2k x-window xul xslt xsl xsd xsb xp xmodem xml xm xl xhtml xdsl x86 wysiwyg www wsdl wormhole worm worksheet workgroup wordperfect wordnet wo wml wlan wireless winzip windowing win32 wiki wi-fi widget whois wep website webmaster webcasting webcam web wdm wav warez wap wan wais wai w3c w3 vxwork vt100 vt vsat vrml vr vpn voip vod vo vm vlsi vldb vlan virtual viewer videotex vhe vhdl vga vdx vcr vax vaporware utf-8 user-friendly usenet usb usability url upload upgrade unzip unix univac unicode uml ukerna uis uim uddi typeface type-ahead twitter tweeter turing tsr truetype trs-80 transputer transistor transceiver touchpad torrent toolbook toolbar toggle tiff thunderbird thumbnail threaded terminal terabyte telnet teletypewriter telepac telenet telecommuting telco tei tcp tcg tc tape $\mathrm{t} 3$ syntax synchronous switching swf svg surfing superjanet superhighway supercomputer subnet string streaming stp stemming stemmer ssl ssi ssh ssd ssadm sql spyware spss spreadsheet spoofing spml spitbol sparc spamming spam som solaris software softcopy socket soa snobol4 snobol sniffer smtp smiley smil smalltalk sm simulation simulate simcity silicon signal shockwave shareware sgml servlet server-side server sequent semiconductor sega sdrc sdlc sdk sdi scsi scrolling screen scanner scalability scada sbus sas sap rw rtf rss rsc rsa rs-232 rs routing router rom robot robohelp risc rgb rfid rfc retrocomputing resolver refactoring recursive real-time $\mathrm{rdl}$ rdf rdbm rdb raster quicktime qbasic python pso pseudocode prototyping prospero prometheus prolog programming programmer procomm processor processing preprocessor prefetch pptp powerpoint postgresql postgre posix porting portal pop3 polynomial-time podcast pod po png plug-in pld playstation plaintext pki pixel pipelining pipelined ping pid photoshop phishing pgp perl perceptron pentium peer-to-peer pdp-11 pdl pdf pcmcia pcm pc paste password parsing 
parser parse palmtop paging packet-switched packet $\mathrm{p} 2 \mathrm{p}$ owl outsourcing ospf oracle opac oop oodbm oodb ooa oo ontology on-line omg oma olap off-line oem odmg odi objectoriented object-orientation oath $02 \mathrm{nvl}$ nt nsfnet non-volatile nintendo nii newsgroup netware netscape netiquette ndl navigator narrowband mysql myspace musl multi-user multithreading multiprocessor multiprocessing multiplexer multimedia msn ms-do mpp mpeg-7 mpeg-4 mpeg-21 mpeg-2 mpeg-1 mpeg mp3 mozilla mouse motorola motherboard mosaic modem mmorpg mmog minix mining minicomputer mimd middleware midas microsoft microprocessor microcontroller microcomputer microarray menu memex megabyte mdi mda md5 mcp matlab mathematica markup malware mainframe mailbox macromedia macintosh machine lyco lurker Itp lossy lossless logon listserv lisp linux linker Idap Icd laptop lambdamoo kwic kqml knowbot kilobyte kh keyboard kerning kb jsp js jpg jpeg joystick javascript java j2ee itsp itil isp isoc isi isee isdn irl irc ir ipv6 ipv4 ipsec ipl ipad ip intranet interworking interoperability internetworking internet intermedia interlink intercom intel inline ingre infoseek informix infobahn impedance imap imaging imac iis iir ifip ietf ieee idss ids idl ict icq icmp i-case icann ibm hytime hypertext hypertalk hypernew hypermedia hyperlink hypercard hyperbase hvd httpd http html hsm hpl hpcc hp hotmail hotlist hotlink hostname honeywell hex hewlett-packard hensa hdtv hdd hci hashing hash harvest hardware hard-coded hacker hack gzip gui gss-api gss gsm gsi gs groupwise groupware gray-scale gpss gpr gpl gopher google gnu gnome gis gigabyte gigabit gif ghostscript fujitsu ftp freeware freenet freebsd framemaker fpga fortran font foaf floppy floating-point flaming flame firmware firewire firewall firefox filemaker fedora faq $f 2 f$ e-zine extranet executable event-driven euronet etl ethernet e-text esim escrow erp ercim epss encryption encoder encode emulator emoticon emc e-mail emac e-learning eisa egp eftpo eft e-commerce ecm ecash ebcdic e2e dynix dvd-rom dvd-r dvd dtp dtd dsssl dss dsl dsdm droid drm dpp dpn dpi downloading download dongle dogpile dn dml dlm dll dle diskette disk disc disa dipp diode digital digerati dicom dialup dhtml dfd desktop decstation decryption debugging debug ddo $\mathrm{ddm} \mathrm{dbms} \mathrm{dbm}$ dbase $\mathrm{dba} \mathrm{db}$ dataview datatrieve database dat darpa dad dac cyber-squatting cyberspace cyberpunk cybernetic cyber cwis cuseeme cursor csv css cscw cs cryptology cryptography cryptanalysis crt crosstalk crossplatform crawler cpu courseware corc corba cookie console computing computer computational computable compuserve compiler commodore coldfusion codec cobol cnn cnet cnc cmo cmd cmc clv client-server click cli cjk cir ciphertext chunker chip checksum chatterbot cgi cello cd-rw cd-rom cd-rewritable cdl cd-i cd ccitt cbt cbr cbir cbd cbasic cav captcha capacitor calculator calc cais cad cacm cache byte button b-tree bsd browser broadband bpel bounce bot bootstrap booting bookmark bluetooth blog bitwise bittorrent bitnet bitmap bioinformatic binarie bibtex beta bebo beamer bcpl baud bandwidth backend babbage avatar av automaton automation automata autocad atm asynchronous assembler asr asdl ascii arpanet arpa archie applet apple apm api apache aol antivirus aml algorithm alc ajax aida adware adsl adl adabas activex acrobat acorn aco acm acl acis $4 \mathrm{gl} 486$ kerberos online inspec computer-aided electronic e-government e-access 